\title{
ApoE and S-100 expression and its significance in the brain tissue of rats with focal contusion
}

\author{
Z.L. Wang ${ }^{1 *}$, R.F. Chai ${ }^{2 *}$, W.S. Yang ${ }^{3}$, Y. Liu ${ }^{1}$, H. Qin ${ }^{1}$, H. Wu ${ }^{1}$, X.F. Zhu ${ }^{1}$, \\ Y.X. Wang ${ }^{1}$ and G. Dangmurenjiafu ${ }^{1}$ \\ 1'Department of Neurosurgery, The First Affiliated Hospital of Xinjiang Medical \\ University, Urumuqi, Xinjiang Province, China \\ ${ }^{2}$ Critical Care Medicine Department, the First Affiliated Hospital of Xinjiang Medical \\ University, Urumuqi, Xinjiang Province, China \\ ${ }^{3}$ Department of Pathology, Successful Hospital Affiliated to Xiamen University, \\ Xiamen, Fujian Province, China \\ *These authors contributed equally to this study. \\ Corresponding author: G. Dangmurenjiafu \\ E-mail: coronary_1_lab@126.com \\ Genet. Mol. Res. 14 (4): 19275-19281 (2015) \\ Received August 9, 2015 \\ Accepted October 18, 2015 \\ Published December 29, 2015 \\ DOI http://dx.doi.org/10.4238/2015.December.29.37
}

ABSTRACT. This study explored the effect of focal cerebral contusion on the expression of ApoE and S-100, and its significance in determining the time of brain injury. Based on a rat model of cerebral contusion, immunohistochemistry was used to analyze the expressions of S-100 and ApoE at different time points after injury. Thirty minutes following cerebral contusion, ApoE protein expression was significantly increased in cortex neurons $(P<0.01)$, and $S-100$ protein expression was significantly $(P<$ 0.001 ) elevated $2 \mathrm{~h}$ after cerebral contusion. Over time, the number of ApoE and S-100 positively expressing cells gradually increased. Three days after injury, ApoE was widely distributed throughout the tissue and the number of ApoE-positive cells and staining intensity reached a peak. ApoE expression decreased after this time point. Five days after cerebral 
contusion, the number of S-100-positive cells reached a peak level of expression higher than that in the control group. Our data demonstrate that the expression of ApoE and S-100 correlated with the progression of focal cerebral contusion. This suggests that both proteins may serve as effective biomarkers of focal cerebral contusions.

Key words: Cerebral contusion; Injury time; S-100; ApoE

\section{INTRODUCTION}

The brain is a vital organ in the human body that is particularly vulnerable to violent injury due to a low elasticity modulus, a weak resistance to differences in physical stress, and a high incompressibility (Zhang, 2002). After serious injury, the morphology and function of the brain is often changed. This includes the altered expression of a variety of biologically active proteins, in a timedependent manner, within neurons and glial cells. Monitoring these changes in gene expression may be useful in determining the time of cerebral contusion. In this study, a rat brain contusion model was established using the free-fall impact method, and immunohistochemical staining (streptavidin-peroxidase method, SP) was used to detect ApoE and S-100 protein expression 10 days following brain injury. Double-positive cells were also recorded to explore the possible mechanism of these changes in a time-dependent manner. These data provide experimental evidence for forensic identification, and clinical diagnosis and treatment of brain injury.

\section{MATERIAL AND METHODS}

\section{Experimental animals and grouping}

Fifty-five adult Wistar rats (both males and females), weighing between 200 and 300 g, were purchased from Xinjiang Medical University Experimental Animal Center. The rats were randomly divided into 2 groups: 1) the 'injury' group with 45 rats, and 2) the 'control' group with 10 rats. The injury group was divided into nine subgroups: $0.5,2,26,12,24 \mathrm{~h}$, and 3, 5, 7, and 10 days. Each subgroup consisted of five rats. The control group was divided into 2 subgroups: the normal control and sham subgroup, with five rats each.

\section{Rat cerebral contusion model}

According to the Feeney's method (Feeney et al., 1981) and previous reports (Wu et al., 2005), a rat cerebral contusion model was established. Specifically, after weighing, the rats were anesthetized by intraperitoneal injection with $0.4 \%$ sodium pentobarbital $(1 \mathrm{~mL} / \mathrm{kg}$ body weight). After fixing the head, an incision was made at the top of the scalp (in the middle), and a circular bone window ( $5 \mathrm{~mm}$ in diameter) was made by dental drill at the position $2 \mathrm{~mm}$ behind the coronal suture and $2 \mathrm{~mm}$ to the right of the sagittal suture while the dura was kept intact. A $50 \mathrm{~g}$ weight was released to free-fall from a height of $20 \mathrm{~cm}$ and hit the bottom of the blunt screw, which impacted the brain causing a partial contusion in the right parietal lobe. Under no conditions were these experiments fatal to the animals. During this procedure, the animals did not die. The bone window was then closed with bone wax, and the wound was disinfected layer by layer to prevent infection. The scalp was sutured and the animal was kept in cages and allowed to feed ad libitum. The rats 
were decapitated and sacrificed at $0.5,2,6,12$, and $24 \mathrm{~h}$, and $3,5,7$, and 10 days after the injury. The skulls of the rats in the sham group underwent drilling but were not subjected to cerebral contusion by the falling weight. The sham group rats were sacrificed $1 \mathrm{~h}$ after drilling. The normal control rats received no treatment and were also decapitated and sacrificed.

\section{Sampling}

After the rats were sacrificed, the whole brain was immediately dissected, washed with saline, and placed in $4 \%$ paraformaldehyde solution at $4^{\circ} \mathrm{C}$ for $24 \mathrm{~h}$. The tissue approximately 1 $\mathrm{mm}$ away from the focal cerebral contusion position, on both sides, was discarded. The central part of the tissue was embedded in paraffin and subjected to $5 \mu \mathrm{m}$ sectioning and HE staining.

\section{Immunohistochemical staining}

The SP immunohistochemistry method was used to detect the expression of ApoE and S-100. Rabbit polyclonal anti-ApoE antibody was used to stain ApoE, with the polyclonal antibody visualized via the SP secondary antibody kit (Beijing Biosynthesis). S-100 was stained using the rabbit anti-S-100 polyclonal antibody (Beijing Zhongshan) along with the DAB chromogenic reagent kit (Tiangen). The procedure was performed in accordance with manufacturer specifications. A PBS solution without any antibody was used as a negative control.

\section{Criteria for immunohistochemical staining}

The neurons or glia with a brown granule in the nuclei or cytoplasm were regarded as positive, while those with blue nuclei and cytoplasm were regarded as negative. The Motic Image Analysis System was adopted to analyze five fields around the focal cerebral contusion in the right parietal cortex (40X, 10-fold). The immunohistochemical staining was used for semi-quantitative detection, and the positive cells were counted.

\section{Statistical analysis}

Data are reported as means \pm standard deviation (SD) and analyzed by the SPSS15.0 software. The number of positive cells in each group was compared using one-way analysis of variance (ANOVA) and the $-\chi^{2}$ test, and the test level was $\alpha=0.05$. A P value less than 0.05 was considered to be statistically significant.

\section{RESULTS}

\section{General results of experimental animals}

While trying to generate the rat cerebral contusion model, five rats died due to respiratory depression after anesthesia, or herniation after cerebral contusion. These dead animals were excluded from the group, and the vacancies were filled by new rats. After cerebral contusion, most rats in the experimental group had a brief coma, were shaggy, and in a head-down curled shape position. The rats regained consciousness 12-16 $\mathrm{h}$ after surgery, and were eating independently. The sham rats were awake 4-6 $\mathrm{h}$ after surgery. 


\section{HE staining}

All rats in each injury group had subdural hematomas, subarachnoid hemorrhages, cerebral edema, and varying degrees of superficial brain tissue setback, broken bones, and bleeding (Figure 1A). At the early stages following cerebral contusion, there was evidence of neuronal swelling, a reduction in neurite outgrowth, dark staining nuclei, blurred prominent nucleoli, and glial cell swelling. With prolonged injury, the contusion area and surrounding neurons were reduced and distorted, and cytoplasmic Nissl bodies had disappeared, which could indicate the progressive development of neuronal necrosis. The swelling of glial cells was more prominent along with hyperplasia. Rats in the sham group had only mild congestion and edema.
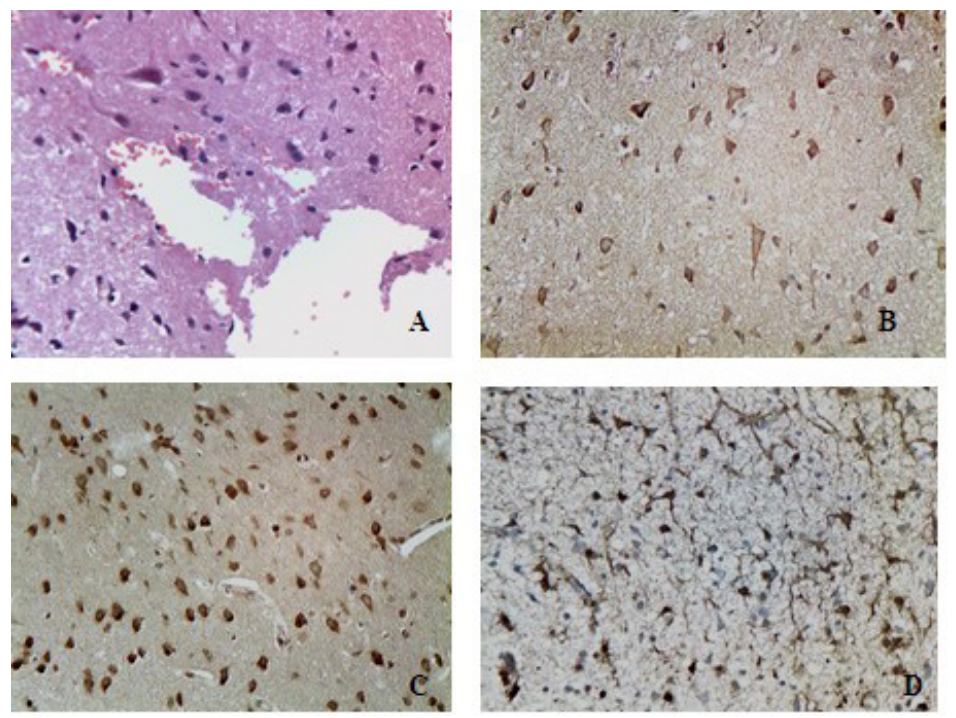

Figure 1. HE staining and ApoE and S-100 expression. A. Superficial brain tissue was broken, with bleeding and tissue edema at $12 \mathrm{~h}$ after cerebral contusion (HE 400X). B. Small number of weak cytoplasm ApoE-positive neurons $30 \mathrm{~min}$ after cerebral contusion (ApoE 400X). C. Large number of strong ApoE-positive neurons and astrocytes 3 days after cerebral contusion (ApoE 400X). D. Large number of S-100-positive astrocytes 5 days after cerebral contusion where expression had peaked (S-100 400X).

\section{ApoE and S-100 expression}

\section{ApoE staining}

A few neurons and glial cells in the cerebral cortex had weak expression of ApoE in the normal control group and the sham group. Thirty minutes after injury, light brown particles could be seen in cytoplasm of a small number of neurons around the cortical contusion foci (Figure 1B). The positive cell number gradually increased from $2 \mathrm{~h}$ to 3 days after injury. There were a large number of neurons and astrocytes that had dark brown granules in the cytoplasm (Figure 1C). ApoE expression began to decline after day 3; however, positively stained neurons and ASTs were still observed 10 days after cerebral contusion. 


\section{S-100 staining}

Some glial cells weakly expressed S-100 in the nucleus and cytoplasm in the normal control and the sham group, but not in neurons. The number S-100-positive cells increased from $30 \mathrm{~min}$ to $24 \mathrm{~h}$ after injury. The number of positively stained cells around the cortical contusion foci was elevated and stronger. The 3 and 5 day post-injury groups showed evidence of a large number of glial cells, predominantly ASTs, with abundant cytoplasm and thickened neuritis (Figure 1D). The S-100 positive cell staining intensity began to decline 7 days after cerebral contusion, but was still detectable in the 10 day group.

\section{Statistical results}

There were statistically significant differences in the number of ApoE and S-100 positively expressing cells in the injury group as compared to the control group. The number of ApoE-positive cells in the 30 min group was significantly higher than that in the control group $(P<0.01)$. Similarly, the S-100-positive cells were significantly elevated $2 \mathrm{~h}$ after injury $(P<0.01)$. Over time, the number of ApoE and S-100 positively expressing cells increased. ApoE-positive cells peaked after 3 days post-injury, and this was maintained until day 5 where the positive cells declined significantly. The number of S-100-positive cells peaked after 5 days post-injury, after which the number of positive cells began to decline. The expression of both proteins was significantly higher than those in the control group 10 days post-injury ( $P<0.01$; Table 1$)$.

Table 1. Number of ApoE and S-100-positive cells after cerebral contusion (means \pm standard error of the mean).

\begin{tabular}{|c|c|c|}
\hline Group & ApoE & S-100 \\
\hline Control & $14.40 \pm 1.14$ & $11.00 \pm 1.58$ \\
\hline $0.5 \mathrm{~h}$ after injury & $29.60 \pm 1.52^{\star \star}$ & $14.00 \pm 1.58^{*}$ \\
\hline $2 \mathrm{~h}$ after injury & $30.00 \pm 1.58^{* *}$ & $19.00 \pm 2.24^{\star * \Delta}$ \\
\hline $6 \mathrm{~h}$ after injury & $33.20 \pm 2.17^{\star *}$ & $25.00 \pm 2.00^{* * \Delta}$ \\
\hline $12 \mathrm{~h}$ after injury & $49.00 \pm 2.74^{\star \star \Delta}$ & $26.80 \pm 1.92^{\star *}$ \\
\hline $24 \mathrm{~h}$ after injury & $60.40 \pm 2.30^{\star \star \Delta}$ & $28.60 \pm 1.52^{* *}$ \\
\hline 3 days after injury & $70.60 \pm 1.14^{\star \star \Delta}$ & $34.00 \pm 1.58^{* \star \Delta}$ \\
\hline 5 days after injury & $64.60 \pm 4.39^{\star *}$ & $39.00 \pm 1.58^{* *}$ \\
\hline 7 days after injury & $54.00 \pm 5.29^{* \star \Delta}$ & $26.40 \pm 2.88^{* \star \Delta}$ \\
\hline 10 days after injury & $44.40 \pm 3.65^{\star \star \Delta}$ & $25.80 \pm 1.64^{\star \star}$ \\
\hline
\end{tabular}

${ }^{* *} \mathrm{P}<0.05$ vs control group; $\triangle \mathrm{P}<0.01$ vs the neighboring group.

\section{DISCUSSION}

In this study, Feeney's method was used to produce a right parietal bone window as the location of cerebral contusion. This area was chosen in order to avoid variability in skull thickness and to ensure the success of the rat focal cerebral contusion model. To get close to the actual prosecution forensic case, we increased the observation period post-injury in the experimental groups. The experimental results showed different degrees of bleeding in rats of all brain injury groups, and varying severity of frustration and necrosis. Some cases displayed evidence of brain tissue tearing and mixed contusions around the incision due to the direct impact upon the dura and brain tissue that lacked protection from the scalp.

ApoE is a type of plasma apolipoprotein that is synthesized primarily in the liver and nervous system. In the latter case, ApoE is synthesized by macrophages and AST in the central 
nervous system (CNS) and peripheral nervous system, respectively. After brain injury, ASTs take up presynaptic cholesterol and phospholipid-rich glial, leading to cholesterol accumulation and large-scale ApoE synthesis. ApoE then forms a non-polar molecule complex with cholesterol, and is taken up by the neuronal membrane surface of very low-density lipoprotein (VLDL) receptor, into regional or distal dendrites of neurons, occur for the regeneration of the cytoplasm and synapses (Liu et al., 2005). Thirty minutes after cerebral contusion, neurons within the cortical contusion foci had significant but weak staining of ApoE compared to the controls $(P<0.01)$, which is consistent with a previous study by Horsburgh et al. (1997). The data were earlier than Orihara's report (Orihara and Nakasomo, 2002), which could be due to species differences, and rats were less tolerated than human. The number and staining of ApoE-positive cells in the day 3 group, following cerebral contusion, had peaked and are predominantly neurons. This level of staining occurred later than in a previous study by Iwata et al. (2005), and may be due to the different animal model used. The 5 day post-injury group showed some evidence of positively stained cells, indicating that the immune response was still high prior to a gradual decline in staining over time.

$\mathrm{S}-100$ is an acidic calcium-binding protein that is mainly secreted by the Schwann cells to the peripheral nervous system. S-100 is predominantly synthesized and secreted by glial cells, particularly ASTs, into the CNS and is an intermediary substance for the glial cells and neurons. Whitaker-Azmitia et al. (1997) reported that S-100 has trophic effects on glial cells and neurons. Indeed, during brain development and recovery from injury, S-100 acts as a neurotrophic factor upon neurons and the surrounding environment. Additionally, S-100 expression in the brain has a protective effect on the brain. During brain damage, there is typically dysfunction in a nerve cell membrane transporter along with impaired mitochondrial respiration (Whitaker-Azmitia et al., 1997). This results in intracellular calcium accumulation. The carboxyl terminus of the S-100 subunit contains an EFhand calcium-binding domain, which undergoes a conformational change when bound to a calcium ion and exposes binding sites that allow S-100 to exert its biological effects.

Two hours following cerebral contusion, S-100 positively stained cells were significantly increased within the contusion foci $(P<0.01)$. This increase developed earlier than in previous reports by $\mathrm{He}$ et al. (2007), which may be due to a difference in the falling body. Similarly, after 5 days post-injury, the number of S-100-positive ASTs increased in the area surrounding the injury. Moreover, neuritis was prominently observed at this time, which is consistent with a previous study.

S-100 is a known marker for AST activation. In various brain injuries, the proliferation and activation of reactive ASTs produce a large amount of S-100, which in turn stimulates further AST proliferation. As a result, a positive feedback effect is created. In this experiment, in 3 and 5 day post-injury groups, the peak expression of the two proteins were substantially overlapping (i.e., the peak ApoE-positive neurons and S-100-positive glial cells overlapped). These data suggest that via the proliferation or release of growth and nutritional factors, a large number of glial cells were involved in the repair of damaged neurons or nerve tissue.

In this study, the expression of ApoE and S-100 is time-dependent, with positive reactions tending to increase, peak, and then decline. The expression peak for the two proteins was 3-5 days after injury, and the peaks of these two proteins had a significant time overlap. Thus, we used two indicators to make comprehensive analysis feasible. Approximately 30 min after cerebral contusion, the expression of ApoE and S-100 around the contusion foci was altered. This suggests that ApoE and S-100 are both sensitive indicators of early head injury. It should be noted that this is an animal study with a limited sample size. Larger studies are required for a more in-depth study along with the use of human brain tissue samples. 


\section{Conflicts of interest}

The authors declare no conflict of interest.

\section{REFERENCES}

Feeney DM, Boyeson MG, Linn RT, Murray HM, et al. (1981). Responses to cortical injury: I. Methodology and local effects of contusions in the rat. Brain Res. 211: 67-77.

He F, Zhang L, Liu Z, Wang S, et al. (2007). The expressions of GFAP and S100 and estimation of post-traumatic intervals after severe brain injury in rats. J. Forensic Sci. 23: 181-184.

Horsburgh K, Fitzpatrick M, Nilsen M and Nicoll JA (1997). Marked alterations cellular localisation and levels of apolipoprotein E following acute subdural haematoma in rat. Brain Res. 763: 103-110.

Iwata A, Browne KD, Chen XH, Yuguchi T, et al. (2005). Traumatic brain injury induces biphasic upregulation of ApoE and ApoJ protein in rats. J. Neurosci. Res. 82: 103-114.

Liu Y, Huang P, He X, et al. (2005). The research and utilization of ApoE on brain injury. J. Law Med. 14: 74.

Orihara $Y$ and Nakasono I (2002). Induction of apolipoprotein $E$ after traumatic brain injury in forensic autopsy cases. Int. J. Legal Med. 116: 92-98.

Whitaker-Azmitia PM, Wingate M, Borella A, Gerlai R, et al. (1997). Transgenic mice overexpressing the neurotrophic factor S-100 beta show neuronal cytoskeletal and behavioral signs of altered aging processes: implications for Alzheimer's disease and Down's syndrome. Brain Res. 776: 51-60.

Wu X, Wang B, Zhang G, et al. (2005). Animal model of graded impact brain injury in rats. Chinese J. Forensic Med. 20: 1-3. Zhang Y (2002). Research progress of brain injury in forensic medicine (I). Chin. J. Forensic Med. 17: 51. 